\title{
Ephrin-B2 paces neuronal production in the developing neocortex
}

\author{
Anthony Kischel, Christophe Audouard, Mohamad-Ali Fawal and Alice Davy * (D)
}

\begin{abstract}
Background: During mammalian cerebral cortex development, different types of projection neurons are produced in a precise temporal order and in stereotypical numbers. The mechanisms regulating timely generation of neocortex projection neurons and ensuring production in sufficient numbers of each neuronal identity are only partially understood.

Results: Here, we show that ephrin-B2, a member of the Eph:ephrin cell-to-cell communication pathway, sets the neurogenic tempo in the neocortex. Indeed, conditional mutant embryos for ephrin-B2 exhibit a transient delay in neurogenesis and acute stimulation of Eph signaling by in utero injection of synthetic ephrin-B2 led to a transient increase in neuronal production. Using genetic approaches we show that ephrin-B2 acts on neural progenitors to control their differentiation in a juxtacrine manner. Unexpectedly, we observed that perinatal neuron numbers recovered following both loss and gain of ephrin-B2, highlighting the ability of neural progenitors to adapt their behavior to the state of the system in order to produce stereotypical numbers of neurons.
\end{abstract}

Conclusions: Altogether, our data uncover a role for ephrin-B2 in embryonic neurogenesis and emphasize the plasticity of neuronal production in the neocortex.

Keywords: Neurogenesis, Neural progenitors, Neocortex, Eph:ephrin signaling, Mouse

\section{Background}

It is estimated that the mouse brain is composed of about 70 million neurons [1], the vast majority of which are generated during fetal life. These neurons come in dozens of different flavors, each neuronal subtype being characterized by wiring partners, location, morphology, neurotransmitter production. The mechanisms ensuring that neuron types and numbers are faithfully produced are only partly understood. Over the years this question has been largely studied in the neocortex, a mammalianspecific region of the brain which is the seat of complex cognitive functions $[2,3]$.

Neurogenesis in the neocortex is characterized by the orderly production of different types of projection

\footnotetext{
*Correspondence: alice.davy@univ-tlse3.fr

Centre de Biologie Intégrative (CBI), Centre de Biologie du Développement (CBD), Université de Toulouse, CNRS, UPS, 118 route de Narbonne, 31062 Toulouse, France
}

neurons that migrate and settle at precise spatial positions, thus eventually forming a six-layered structure $[2$, 3]. These neurons can be grouped in two main classes: early born deep layer (DL) neurons which project to subcortical targets and late born neurons that extend inter-hemispheric projections, mostly located in the upper layers (UL) of the neocortex [4]. Both DL and UL neurons are born from neural progenitors (NP) located in the germinal zones of the neocortex, namely the ventricular zone (VZ) and the subventricular zone (SVZ) [5-7]. At the origin of these NP are neuroepithelial cells that actively proliferate to amplify the initial pool of NP and then transition to the apical progenitor (AP) fate. At the onset of neurogenesis there are two main types of $\mathrm{NP}$ in the neocortex: Pax6+ AP whose soma are located in the VZ and Tbr2+ basal progenitors (BP) populating the SVZ. Live imaging and lineage tracing studies in the mouse have shown that asymmetric division of AP give

(c) The Author(s). 2020 Open Access This article is licensed under a Creative Commons Attribution 4.0 International License, which permits use, sharing, adaptation, distribution and reproduction in any medium or format, as long as you give appropriate credit to the original author(s) and the source, provide a link to the Creative Commons licence, and indicate if changes were made. The images or other third party material in this article are included in the article's Creative Commons licence, unless indicated otherwise in a credit line to the material. If material is not included in the article's Creative Commons licence and your intended use is not permitted by statutory regulation or exceeds the permitted use, you will need to obtain permission directly from the copyright holder. To view a copy of this licence, visit http://creativecommons.org/licenses/by/4.0/ The Creative Commons Public Domain Dedication waiver (http://creativecommons.org/publicdomain/zero/1.0/) applies to the data made available in this article, unless otherwise stated in a credit line to the data. 
rise either to neurons (direct neurogenesis) or to $\mathrm{BP}$ which will then terminally divide into neurons (indirect neurogenesis) [8-10]. Using a Tbr2(Cre) mouse line for lineage tracing it was estimated that indirect neurogenesis contribute $67.5 \%$ of projection neurons in the neocortex [11]. More recently, a new type of BP, outer radial glial cells (oRG), has been identified and shown to be massively expanded in the primate neocortex compared to rodents [12]. Because these cells are able to undergo a large number of symmetric proliferative divisions before terminal differentiation, they are believed to be the cellular source for the massive expansion of the neocortex in primates [13].

Time is a key parameter in neocortex development, influencing both the number and the type of neuron generated [14]. Indeed, the duration of the initial NP expansion phase, the rate of NP self-renewal vs. differentiation, as well as the dynamics of NP division are important temporal parameters that have impacts on final neuron numbers. One striking illustration of this is the difference in pace of development that distinguishes mouse and human neocortex development. Neuron production lasts about a week in the mouse vs. 17 weeks in humans and this correlates with an estimated thousand fold increase in neuronal outputs [15]. In addition, timing of differentiation defines the type of neuron produced, with sequential production of DL neurons first and UL neurons last. While mechanisms controlling NP self-renewal vs differentiation have been studied in great detail, mechanisms that are crucial to a timely and orderly neuronal production are less characterized.

Accumulating evidence indicates that progression in the temporal sequence relies on information provided to NP by their local environment. The first observations suggesting that extrinsic information was important to set the pace of neurogenesis in the neocortex came from heterochronic transplantation studies in the ferret showing that environmental cues present at late stages of cortical development within the germinal zone can "re-program" a young NP to give rise to a late-born projection neurons [16]. This question has been reinvestigated with modern techniques recently and it was shown that late AP remain temporally plastic and can adapt to earlier temporal environments by changing their molecular and neurogenic fate [17]. In contrast, late born BP lack this temporal plasticity [17]. Several studies in the mouse have identified extrinsic signals, mainly in the form of feedback from post mitotic neurons, that act on NP to modulate the orderly production of projection neurons [18-22]. More strikingly, it has been recently demonstrated that neuronal loss induced from early to mid corticogenesis is compensated by the over production of UL neurons at late stages of corticogenesis in the mouse [23], indicating that NP are able to sense and respond to environmental cues, adapting their behavior to modulate neuronal production either in types or in numbers.

Eph/ephrin signaling is a bi-directional cell-to-cell communication pathway that plays important roles in tissue patterning [24] but has also been implicated in neurogenesis mainly in the adult brain [25]. In the developing neocortex, previous studies have reported a role for ephrinB1 and EphA4 in maintaining the progenitor fate [26-29] while ephrinA5 and EphA7 were described as pro-apoptotic [30-32]. In vitro and in vivo studies in the adult mouse have shown that one member of the family, ephrin-B2, is a neurogenic cue [33, 34]. For instance, gain and loss of function experiments have shown that ephrinB2 promotes commitment of NSC to the neuronal fate at the expense of the neural stem cell fate in the hippocampus [34]. However, the role of this membrane-bound protein in embryonic neurogenesis in the neocortex has not been reported. Here, we hypothesized that ephrin-B2 could play a role in the timely production of projection neurons in the neocortex. Using gain and loss of function paradigms in vivo we describe a role for ephrin-B2 in pacing neuronal production in the neocortex. At mid-corticogenesis, neuronal numbers are decreased in loss of function conditions and increased in gain of function conditions. Unexpectedly, we show that both in loss and gain of function situations, neuronal numbers are normal at birth.

\section{Results}

\section{Ephrin-B2 is expressed in progenitors and modulates neuronal production}

To address a potential role for ephrin-B2 in controlling neurogenesis in the developing neocortex, we first surveyed its expression in this tissue by in situ hybridization (ISH), from the neuroepithelial stage (E10.5) to the perinatal stage (E18.5) (Fig. 1a). Efnb2 is strongly expressed in neuroepithelial cells at E10.5 and it remains expressed in NP at E13.5. At E13.5, expression of Efnb2 is also detected in the cortical plate (CP), in a high-lateral to lowmedial gradient which coincides with the progression of neurogenesis. At later stages, expression of $E f n b 2$ is low in progenitors and in DL neurons, while high expression is observed in UL neurons. To assess expression of $E f n b 2$ in NP at single cell resolution, we made use of a reporter mouse line that expresses a nuclear GFP under the control of the endogenous Efnb2 promoter [35]. Epifluorescence detection of GFP in thick vibratome sections of the neocortex at E12.5 shows that Efnb2 is expressed in the majority of NP and is strongly upregulated in new born neurons located basally to the VZ (Fig. 1b). Co-immunostaining with an antibody that detects the phosphorylated form of EphB1-3 indicates that these receptors are phosphorylated both in NP and in 


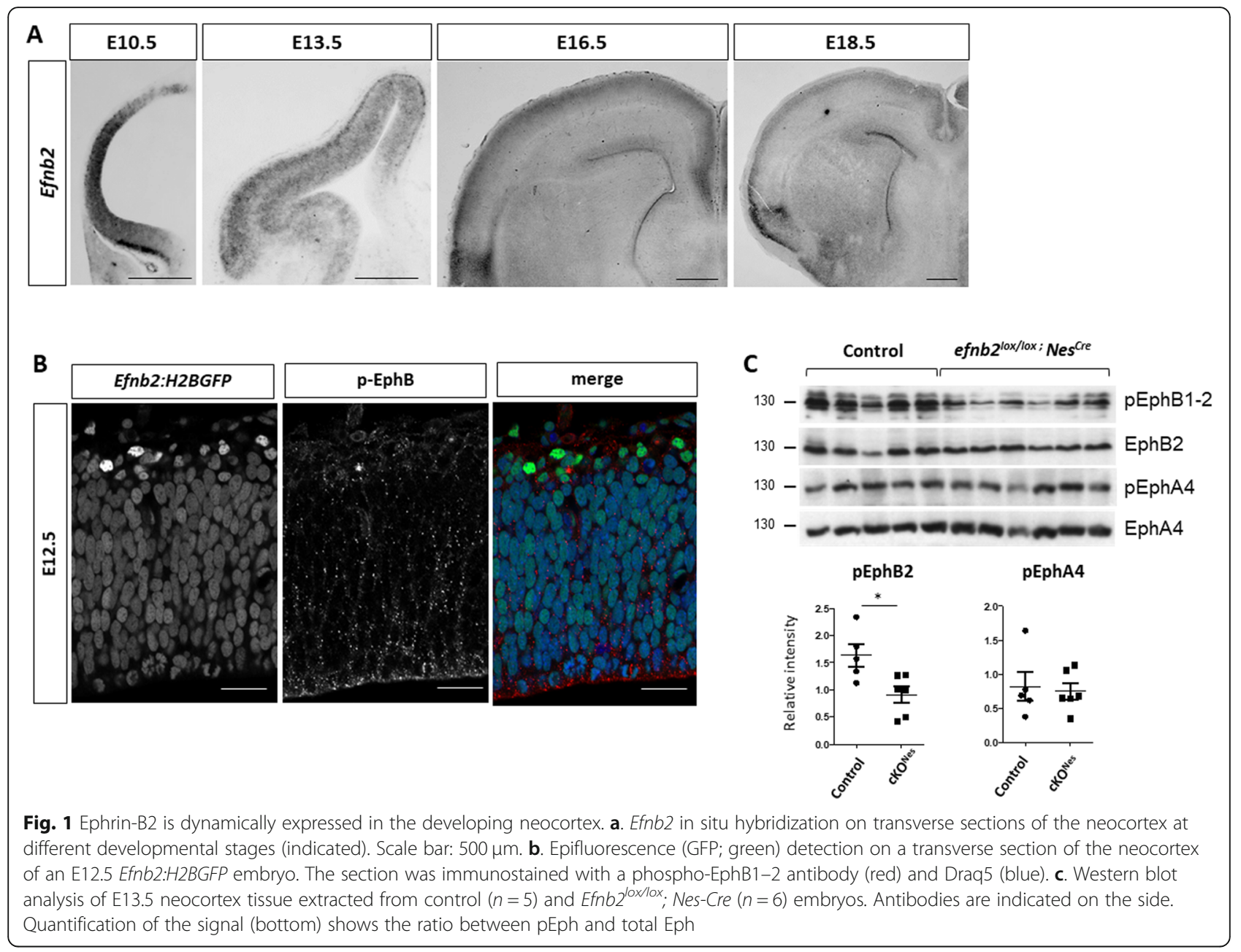

neurons (Fig. 1b) suggesting that EphB:ephrinB2 signaling is active in these cells. To uncover the functional significance of this activation, we generated conditional mutant embryos using Efnb2 ${ }^{\text {floxed }}$ [36] mice and the Nestin-Cre allele [37] which fully excises Efnb2 as early as E11.5 in the neocortex as shown by in situ hybridization (Sup Figure 1A). First, to evaluate the consequence of deleting Efnb2 on Eph:ephrin signaling we monitored the phosphorylation status of EphB1-2 in the neocortex of E13.5 control and Efnb2 $2^{\text {lox/lox }}$; Nestin-Cre $\left(\mathrm{cKO}^{\mathrm{Nes}}\right)$ embryos. Western blot analysis shows that tyrosine phosphorylation of EphB1-2 is decreased in the conditional mutants (Fig. 1c). In parallel, we monitored the phosphorylation status of EphA4, which is also a cognate receptor for ephrin-B2. No change in the phosphorylation status of EphA4 was observed in $\mathrm{CKO}^{\text {Nes }}$ embryos (Fig. 1c). Altogether, these results indicate that loss of ephrinB2 specifically impairs EphB signaling in the developing neocortex.

Next we analyzed neuronal numbers at E16.5 in control and $\mathrm{cKO}^{\mathrm{Nes}}$ embryos using a combination of Tbr1 and Satb2 antibodies that are markers for early born and late born neurons, respectively [38], and DAPI to label all nuclei. Immunostaining and imaging of paraffin sections revealed an apparent decrease in the number of neurons in the $\mathrm{CP}$ of $\mathrm{cKO}^{\mathrm{Nes}}$ embryos compared to controls (Fig. 2a). To quantify this phenotype, we measured the CP thickness (Fig. 2b) and manually counted neuron numbers, including DAPI+ Tbr1- Satb2- neurons, in control and $\mathrm{cKO}^{\text {Nes }}$ embryos, analyzing and averaging 3 distinct regions of interest (ROI) corresponding to medial, dorsal and lateral positions (Fig. 2c). Both parameters were reduced in $\mathrm{cKO}^{\mathrm{Nes}}$ embryos indicating that excision of $E f n b 2$ leads to a reduction in neuron numbers in the neocortex CP. Closer inspection of the data by neuronal marker and by ROI indicated that the reduction in neuron numbers was mostly due to a decrease in Satb2+ neurons and that it followed a mediolateral gradient, with a stronger reduction medially than laterally (Fig. 2d-f). Importantly, the decreased number of neurons in the $\mathrm{CP}$ of $\mathrm{cKO}^{\mathrm{Nes}}$ embryos did not correlate with Satb2+ cells stacked in the intermediate zone, in fact the intermediate zone surface area was reduced (Sup Figure $2 \mathrm{~A}, \mathrm{~B})$, nor was it correlated with an increased number 


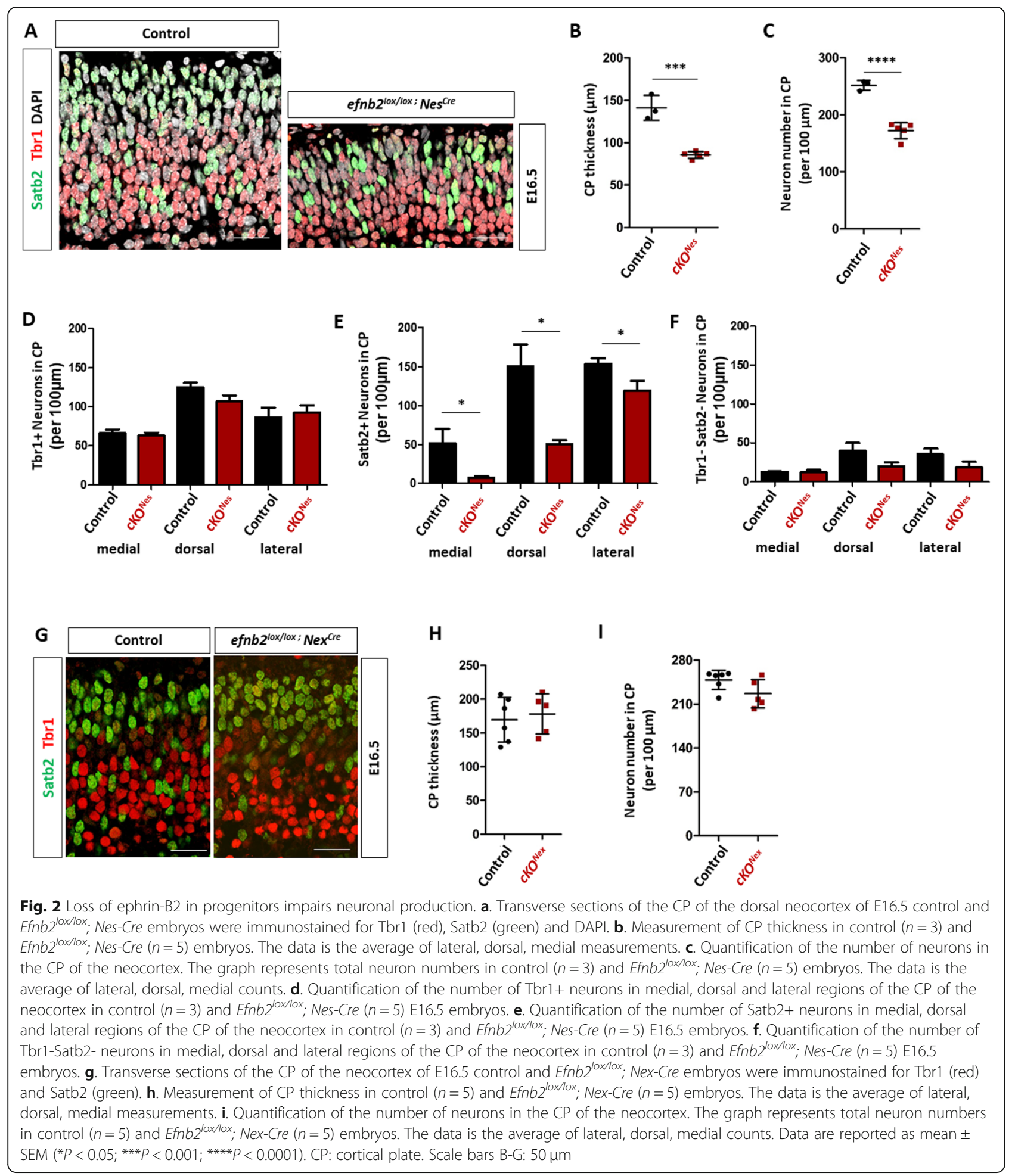

of apoptotic cells (Sup Figure 2C) ruling out cell death or migration defects as potential causes for the observed phenotype.

As described above, Efnb2 is expressed both in neurons and in progenitors and the Nestin-Cre allele eliminates Efnb2 expression in both populations. To ask whether neuronal or progenitor ephrin-B2 expression is required to control neuron numbers, we generated conditional mutant embryos using the Nex-Cre mouse line [39] which excises Efnb2 in neurons but not in progenitors $\left(\mathrm{cKO}^{\mathrm{Nex}}\right)$ as shown by in situ hybridization (Sup Figure 1B). We performed immunostaining with Tbr1 
and Satb2 on paraffin sections of E16.5 embryos (Fig. 2g) and measured the thickness of the $\mathrm{CP}$ and quantified neuronal numbers. No difference in $\mathrm{CP}$ thickness (Fig. 2h) nor in the number of neurons (Fig. 2i) was observed in the $\mathrm{cKO}^{\mathrm{Nex}}$ mutant embryos. Altogether, these results indicate that ephrin-B2 is required in neocortical progenitors to control neuronal production, with a strong effect on Satb2+ neurons.

\section{The decrease in neuron numbers in $\mathrm{CKO}^{\mathrm{Nes}}$ embryos is transient}

The fact that the reduction in neuron numbers followed a mediolateral gradient at E16.5, which also corresponds to the neurogenic gradient, hinted at a dynamic evolution of the phenotype. Hence, to better characterize the phenotype observed in $\mathrm{cKO}^{\mathrm{Nes}}$ embryos we quantified $\mathrm{CP}$ thickness and neuron numbers throughout corticogenesis. We performed immunostaining with Tbr1 and Satb2 at two early stages of corticogenesis and 2 perinatal stages (Fig. 3a) and focused on Tbr1+ and Satb2+ neurons. These analyses showed that while $\mathrm{CP}$ thickness is significantly decreased at $\mathrm{E} 16.5$ in $\mathrm{cKO}^{\mathrm{Nes}}$ embryos, by E18.5 the decrease in no longer statistically significant and at P4 there is no detectable difference (Fig. 3b). With respect to neuron numbers, no statistically significant difference was observed in $\mathrm{cKO}^{\mathrm{Nes}}$ at E13.5 and E14.5 but at E16.5 the number of neurons was decreased in $\mathrm{CKO}^{\text {Nes }}$ (Fig. 3c). This decrease is no longer observed at E18.5 and P4 (Fig. 3c). To capture the evolution of neuronal numbers in a dynamic way, and to ask whether Tbr1+ and Satb2+ neurons were equally or differentially affected, we plotted the mean neuronal numbers for each type of neurons at each developmental stages and represented the data as curves (Fig. 3d-f and Sup Figure 3 for scatter plots with error bars). These results clearly show that loss of ephrin-B2 leads to a decrease in neuronal production at mid-stages of corticogenesis that is compensated at late stages of neocortex development. No statistically significant change was observed for Tbr1+ neurons or Tbr1-/Satb2- neurons at any stage (Sup Figure 3F). In contrast, Satb2+ neurons are severely diminished at E16.5 and their number is compensated at E18.5 (Fig. 3f and Sup Figure 3C, D). Altogether, this data indicates that loss of ephrin-B2 transiently impairs the production of Satb2+ neurons and that it is not due to a switch in neuron identity.

\section{Decreased neuron numbers in $\mathrm{cKO}^{\mathrm{Nes}}$ embryos correlate with slight changes in progenitor numbers}

Decreased neuronal production could be caused by various processes affecting progenitors such as changes in cell cycle duration, modifications in progenitor numbers, imbalance in self-renewal vs. differentiation. To discriminate between these possible causes, we performed double EdU/BrdU labelling as previously described [40] to estimate progenitor cell cycle length and to quantify progenitor populations. To capture progenitor cell cycle length we combined EdU/BrdU immunostaining with Tbr2 immunostaining and considered that Tbr2+ cells are $\mathrm{BP}$ and Tbr2- cells in the VZ are AP (Fig. 4a). We manually counted the number of progenitors (Tbr2- VZ cells and Tbr2+ cells called $\mathrm{P}$ cells), the number of EdU+ P cells (called L cells) and the number of EdU + BrdU+ $P$ cells (called $S$ cells) in control and $\mathrm{cKO}^{\mathrm{Nes}}$ E13.5 embryos (see also Methods and Sup Figure 4A, B). These numbers were used to estimate cell cycle length as described previously [40]. No difference in cell cycle length was observed between control and $\mathrm{cKO}^{\mathrm{Nes}} \mathrm{em}$ bryos when analyzing AP and BP progenitors together (Fig. 4b) or separately (Sup Figure 4C). Next, we reanalyzed the data to quantify numbers of each type of progenitors in both genotypes. With this analysis we detected a slight but statistically significant increase in the total number of progenitors in $\mathrm{cKO}^{\mathrm{Nes}}$ embryos which does not appear to be restricted to one type of progenitors (Fig. 4c). A similar trend was observed at E14.5 without reaching statistical significance (Fig. 4d, e). These data suggest that modification in cell cycle length is unlikely to account for the neuronal phenotype observed in $\mathrm{CKO}^{\mathrm{Nes}}$ embryos. Instead, these results suggest that loss of ephrin-B2 could impair the differentiation process and favor the progenitor fate.

\section{Injection of eB2-fc in lateral ventricles leads to a transient increase in neuronal production}

To test whether ephrin-B2 directly acts as a signal to promote neuronal differentiation (and thus neuronal production), we injected clustered recombinant ephrin-B2-Fc protein (eB2-Fc) or EphB2-Fc protein into the lateral ventricles of developing E13.5 embryos to artificially (and transiently) activate Eph forward or ephrin reverse signaling, respectively. Injected brains were collected at E15.5 and E18.5 and tissue sections were immunostained with Tbr1 and Satb2 antibodies (Fig. 5a). Quantification of neuron numbers showed that a single injection of eB2-Fc (but not EphB2-Fc; Sup Figure 5) leads to an increased number of neurons at E15.5 compared to control injected samples (Fig. 5b). Interestingly, at E18.5 control and eB2-Fc injected samples had similar numbers of neurons (Fig. 5b) indicating that the tissue is able to adapt to either too few or too many neurons. Detailed examination of the data by neuron populations reveal that Satb2+ neurons account for most of the increase in neuronal production at E15.5 (Fig. 5c), again suggesting that the production of this population of neurons is highly sensitive to the ephrin-B2 signal. The increased neuronal production at E15.5 correlated with 

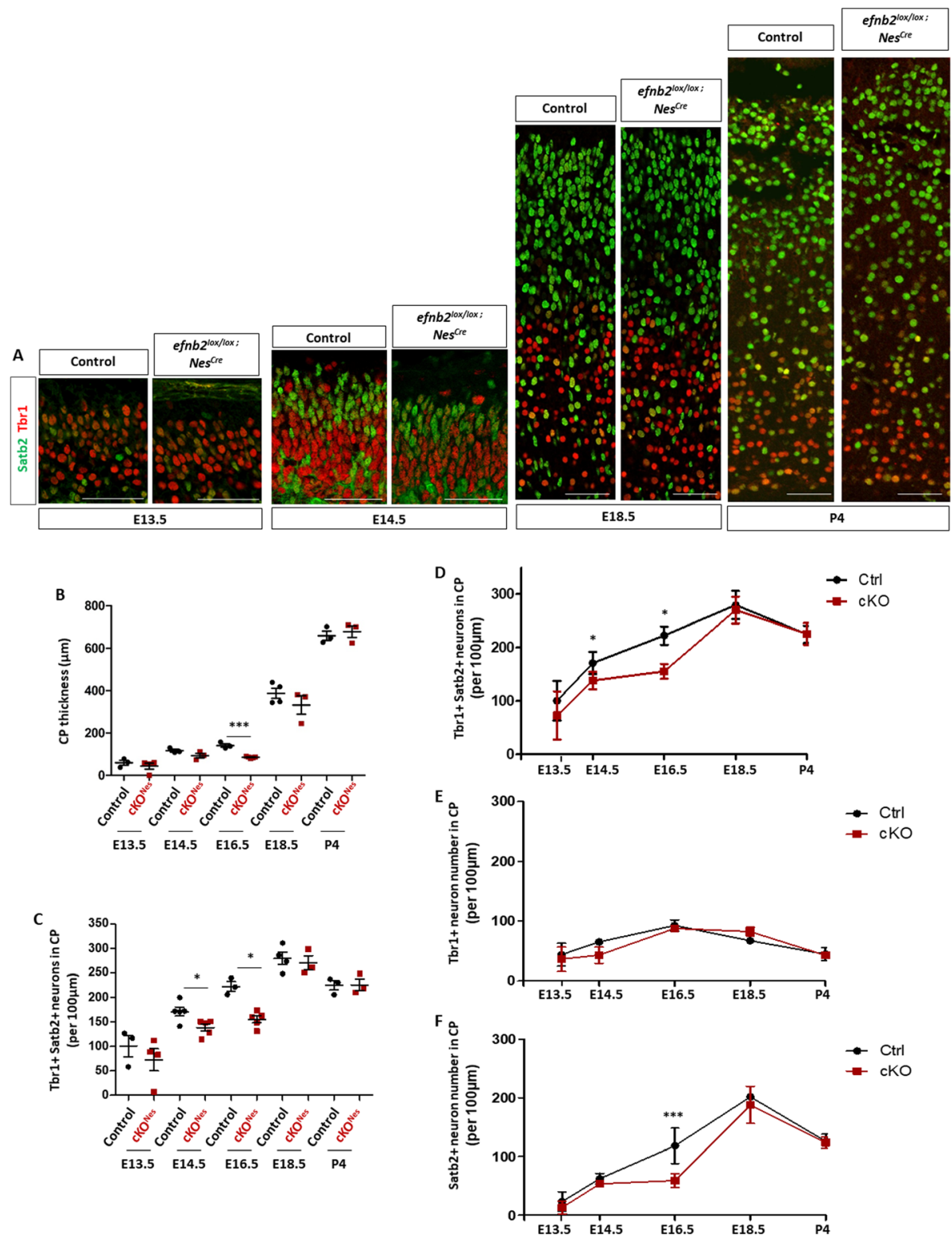

Fig. 3 The decrease in neuronal number in the neocortex of Efnb2 $\mathrm{CKO}^{\text {Nes }}$ is transient. a. Transverse sections of the CP of the neocortex of

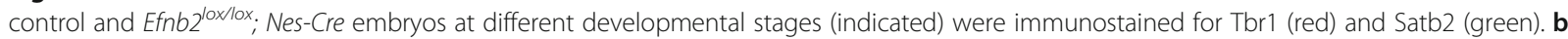
Measurement of CP thickness in control ( $n=3$ or 4$)$ and Efnb2 $2^{\text {loxlox }}$; Nes-Cre ( $n=3$ or 4$)$ embryos at all stages. c. Quantification of the number of neurons in the CP of the neocortex at all stages. The graph represents the number of Tbr1+ and Satb2+ neurons in control and Efnb2 ${ }^{\text {loxlox; }}$ NesCre embryos. $\mathbf{d}$. Mean total neuron numbers at each developmental stages of both genotypes were plotted on a curve graph. e. Mean numbers of Tbr1+ neurons at each developmental stage were plotted on a curve graph. $\mathbf{f}$. Mean numbers of Satb2+ neurons at each developmental stage were plotted on a curve graph. Data on graphs are reported as mean $\pm \mathrm{SEM}$, unpaired t-test and 1-way ANOVA with Bonferroni's multiple comparison test $\left.{ }^{*} P<0.05 ;{ }^{* *} P<0.001\right)$. CP: cortical plate. Scale bars: $50 \mu \mathrm{m}$

a decreased number of Pax6+ AP in eB2-Fc injected samples (Fig. 5d). These results indicate that transient activation of Eph forward but not ephrin reverse signaling in AP promotes neuronal production and they reveal that the tissue is able to compensate both midcorticogenesis decrease and increase in neuronal numbers.

\section{Discussion}

Ephrin-B2 is a pro-neurogenic cue in the embryonic neocortex

Our study reveals an unappreciated role for ephrin-B2 in controlling neuronal production in the embryonic neocortex. We show that conditional excision of ephrin-B2 leads to a transient reduction in neuronal numbers while 


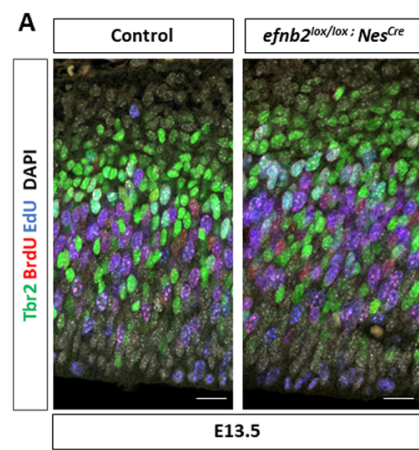

B

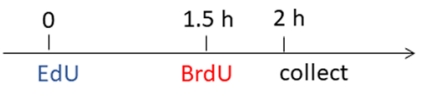

$T_{S}=1.5(L$ cells $/ \mathrm{S}$ cells $)$

$\mathrm{T}_{\mathrm{C}}=\mathrm{T}_{\mathrm{s}} /(\mathrm{S}$ cells $/ \mathrm{P}$ cells $)$

$\mathrm{P}$ cells $=$ Tbr2 ${ }^{-} \mathrm{VZ}$ cells $+\mathrm{Tbr}^{+}$cells

$L$ cells $=E d U^{+} P$ cells

$S$ cells $=E d U^{+}$BrdU $+P$ cells

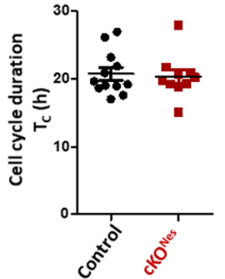

C
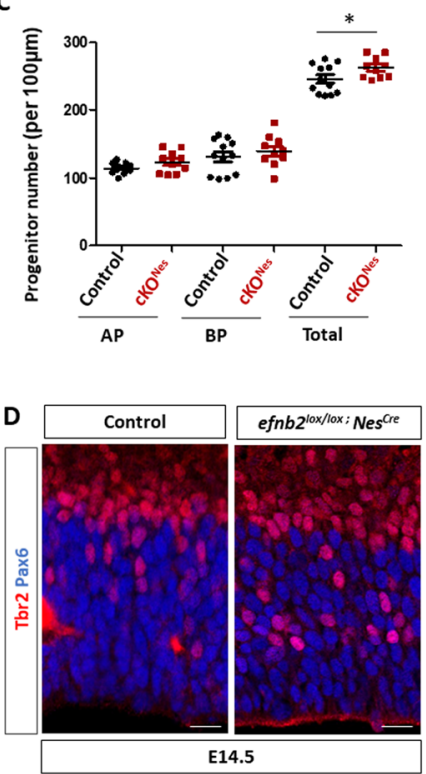

E

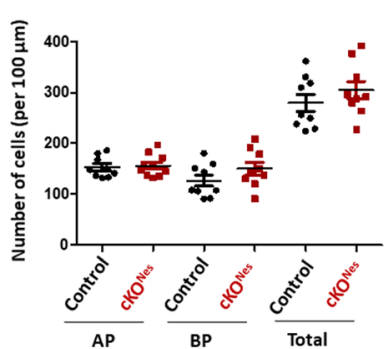

Fig. 4 Cell cycle duration and numbers of progenitors in Efnb2 $\mathrm{CKO}^{\text {Nes }}$. a. Transverse sections of the neocortex of EdU/BrdU-injected E13.5 control

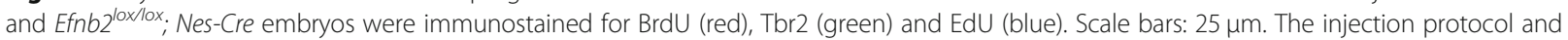
the various cell populations that were counted are indicated on the right. $\mathbf{b}$. S-phase duration $\left(T_{s}\right)$ and cell cycle duration $\left(T_{c}\right)$ were calculated using the indicated formulas. The graph shows $T_{c}$ in control $(n=6)$ and Efnb2 ${ }^{\text {lox/lox }}$; Nes-Cre embryos $(n=5)$. Data for each counted hemisphere are plotted. c. Quantification of AP (Tbr2-), BP (Tbr2+) and total progenitor numbers in control $(n=6)$ and Efnb2 $2^{\text {loxlox }}$; Nes-Cre embryos $(n=5)$. Data for each counted hemisphere and mean \pm SEM are reported, unpaired t-test with Welch's correction $\left({ }^{*} P<0.05\right)$. $d$. Transverse sections of the neocortex of E14.5 control and Efnb2 $2^{\text {lox/lox}}$; Nes-Cre embryos were immunostained for Tbr2 (red) and Pax6 (blue). Scale bars: $25 \mu \mathrm{m}$. e. Quantification of AP (Pax6+), BP (Tbr2+) and total progenitor numbers in control $(n=4)$ and Efnb2 $2^{\text {loxlox; }}$; Nes-Cre embryos $(n=4)$. Mean \pm SEM are reported. AP: apical progenitors; BP: basal progenitors

a single injection of clustered eB2-Fc recombinant protein in lateral ventricles of developing embryos induces a transient increase in neuronal production. This is reminiscent to the role of ephrin-B2 in promoting neurogenesis in the adult hippocampus which has been reported previously [34]. Indeed, hippocampal bilateral injection of clustered eB2-Fc recombinant proteins led to an increased commitment of NSC to the neuronal fate at the expense of the neural stem cell fate while loss of ephrinB2 decreased neurogenesis in the subgranular zone. One interesting aspect of that study was to show that ephrinB2 was expressed and required in astrocytes to control neural stem cell commitment in a juxtacrine manner
[34]. Here, we show that ephrin-B2 is expressed and required in NP but our data does not discriminate between a requirement in AP vs. BP. However, the injection experiments suggest that ephrin-B2 acts on NP in a juxtacrine manner to activate Eph signaling and to promote neurogenesis. Although the gain and loss of function data are consistent in terms of neuronal production, the observed changes at the level of NP are different. In the loss of function experiment, both populations of Tbr2- and Tbr2+ NP appear to be slightly affected while in the gain of function experiment it is the population of Pax6+ AP that is modified. This discrepancy could be due to the fact that AP are primarily targeted in the 

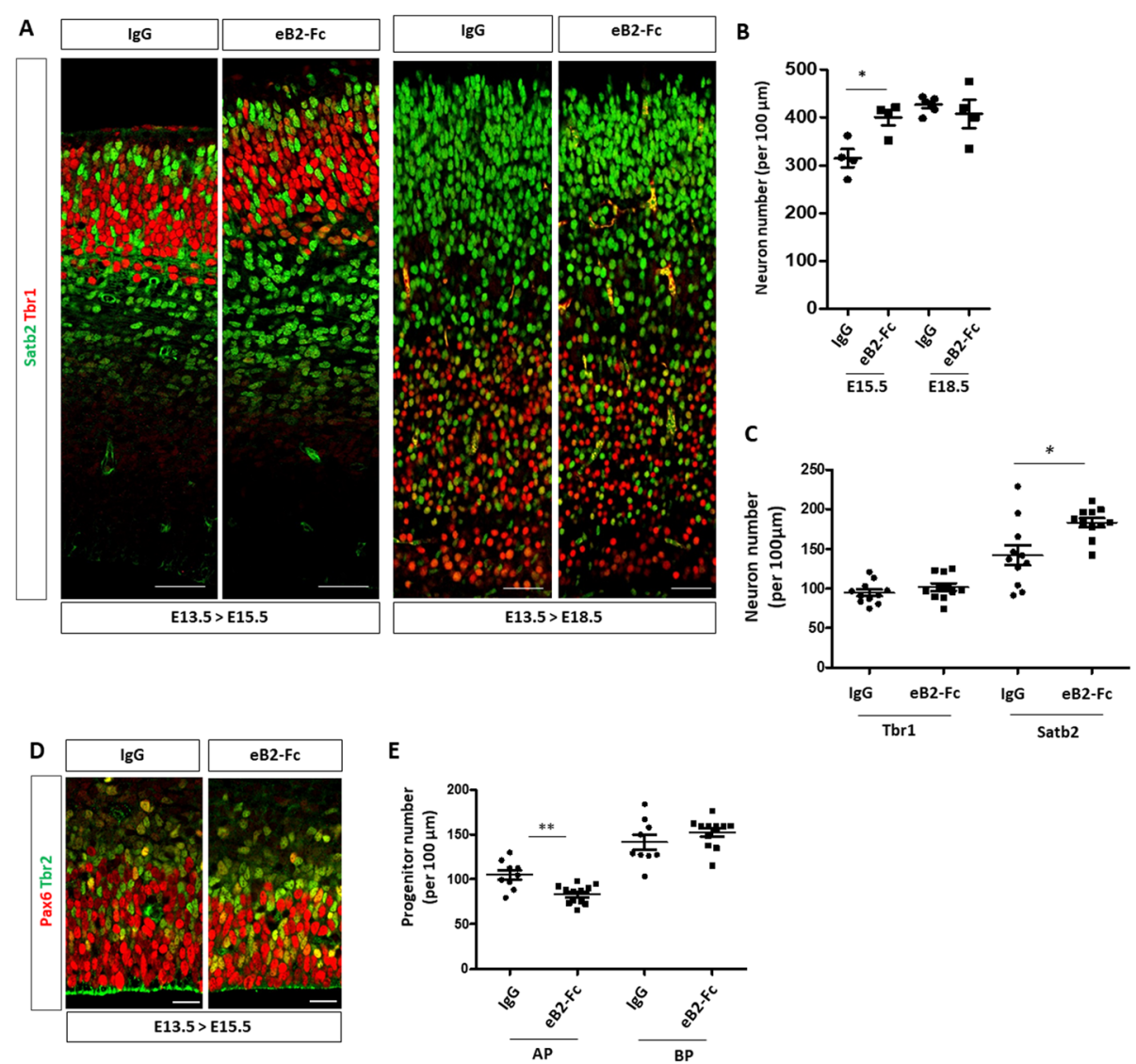

Fig. 5 Acute injection of eB2-Fc in the lateral ventricle leads to an increased neuronal production. a. Transverse sections of the neocortex of E15.5 or E18.5 control injected embryos (IgG) $(n=4)$ and embryos injected with eB2-Fc $(n=4)$ were immunostained for Tbr1 (red) and Satb2 (green). Scale bars: $50 \mu \mathrm{m}$. b. Quantification of the number of neurons in the neocortex of E15.5 and E18.5 embryos. The graph represents numbers of Tbr1+ and Satb2+ neurons in the lateral region of the neocortex in control and eB2-Fc injected embryos. c. Quantification of the number of Tbr1+ and Satb2+ neurons in the neocortex of E15.5 injected embryos (dorsal region of the neocortex). Data for each counted hemisphere are plotted. d. Transverse sections of the ventricular zone and subventricular zone of E15.5 control injected embryos (lgG) and embryos injected with eB2-Fc (dorsal region of the neocortex) were immunostained for Pax6 (red) and Tbr2 (green). Scale bars: $20 \mu m$. e. Quantification of the number of Pax6+ and Tbr2+ progenitors in $\operatorname{lgG}(n=4)$ and eB2-Fc $(n=5)$ injected embryos. Data for each counted hemisphere are plotted. Data are reported as mean \pm SEM. Mann-Whitney statistical test $\left({ }^{*} P<0.05 ;{ }^{* *} P<0.01\right)$

injection paradigm while in the genetic loss of function paradigm, ephrin-B2 is excised from both AP and BP. Of note, we showed recently that a decrease in Sox $2+$ NP and an increase in neuron numbers is also observed following injections of clustered eB1-Fc in lateral ventricles of the developing mouse neocortex [41]. Whether ephrin B1 and ephrin-B2 promote neuronal production via similar mechanisms will have to be explored further.

The onset of neuronal production does not appear to be affected in absence of ephrin-B2, suggesting that the impairment in neuronal production in $E f n b 2 \mathrm{CKO}^{\mathrm{Nes}}$ at mid-corticogenesis is due to a slowing down of the neurogenic process rather than a delay. What could be the cause of this? Based on a similar phenotype observed in Brap mutant embryos, we hypothesized that cell cycle duration might be changed. Indeed, loss of Brap, a RasErk signaling modulator with E3 ligase activity led to a prolonged cell cycle which impeded neuronal production without significantly affecting the number of Pax6+ and Tbr2+ NP [42]. However, using the sequential EdU/ BrdU injection protocol we were not able to detect a difference in cell cycle duration in E13.5 $\mathrm{cKO}^{\mathrm{Nes}}$ embryos, indicating that cell cycle alteration is unlikely to be the cause of the neuronal phenotype. Instead, our data suggest that ephrin-B2 controls the transition from NP to neurons. Indeed, in both loss and gain of function conditions we observed an inverse correlation between progenitor numbers and neuron numbers. In fact, the gain of function data suggests that ephrinB2 may promote direct neurogenesis from Pax6+ AP since the number of Tbr2+ progenitors were unaffected. It is puzzling, however that the observed changes in progenitor numbers were very small compared to changes in neuron numbers. 


\section{A compensatory mechanism normalizes neuron numbers} in the neocortex

The fact that neuronal production is compensated over time in the neocortex of Efnb2 mutants highlights the existence of a compensatory mechanism during corticogenesis. It also suggests that ephrin-B2 is required transiently, at early stages of corticogenesis, a time window that matches the peak of its expression in NP. A similar phenotype has been shown for Caspr (Contactin-associated protein), a member of the Neurexin family of adhesion molecules. Similar to ephrin-B2, Caspr expression peaks at early stages of corticogenesis in the ventricular zone and Caspr mutant exhibit transient decrease in neuronal production which was compensated at E18.5 [43]. The authors proposed that Caspr controlled the timing of NP differentiation by modulating Notch signaling [43]. A similar powerful compensation mechanism was also evidenced recently by transiently inducing neuronal cell death in the neocortex using genetic tools. In this genetic context, the loss of DL neurons was compensated by an over production of UL neurons, which, the authors showed, resulted from the increased proliferation of BP [23]. Altogether, these studies highlight the fact that compensation is possible when early and transient regulatory mechanisms are impacted. On the other hand, many genes that cause autosomal recessive primary microcephaly encode centrosomal proteins that are ubiquitously expressed [44] and this may explain why no compensation is observed when these genes are mutated. Similarly, Brap mutants exhibit microcephaly at P28 [42], indicating that neuronal production is not compensated in these animals, probably because Brap is a ubiquitously-expressed protein whose function is required in NP throughout corticogenesis.

In the future, it would be interesting to identify external signals and intrinsic pathways that allow NP to sense the state of neuronal production and adapt their behavior (e.g. self-renewal vs proliferation vs differentiation). Clearly, our data shows that these compensatory mechanisms are not dependent on ephrin-B2. One candidate could be the neurotrophin Ntf3 which is produced by early born neurons, and acts on NP to promote the switch from AP to BP [19]. It is important to note that while this role of $\mathrm{Ntf3}$ was convincingly described using in utero electroporation experiments, $\mathrm{Ntf3}^{-/-}$mutant neocortex exhibit only a mild neuronal phenotype at E18.5 [19], again suggesting compensatory mechanisms. Another mechanism could be direct Notch signaling from newborn neurons to progenitors via retention of their apical endfoot [22]. Mechanical cues could also represent potential sensing mechanisms. Indeed, tissue crowding or mechanical stretch have been shown to regulate cell proliferation in other contexts [45] and mechanical morphogenesis is now being recognized as a driver of neocortex development and evolution [46]. Another important question that will have to be addressed in the future is whether neurons that are generated in an inappropriate time-window upon compensation are fully functional and correctly integrated into neuronal circuits. This is particularly relevant in the context of human studies which have shown that $E f n B 2$ haploinsufficiency causes neurodevelopmental delay [47].

\section{Conclusions}

Our study adds ephrin-B2 to the list of extrinsic signals that control neurogenesis and it adds embryonic neuronal production to the list of ephrin-regulated functions. Further, it highlights that development of the neocortex is plastic, since this tissue is able to adapt its neuronal output to the state of the system.

\section{Methods \\ Animals}

All experimental animals were generated by breeding at the CBI animal facility according to standard SPF procedures. Efnb2 $2^{\text {H2BGFP }}$, Efnb2 $2^{\text {loxlox }}$, Nestin-Cre and Nex-Cre mouse (Mus musculus) lines have been described previously [35-37, 39]. Efnb2 $2^{H 2 B G F P}$ mice were generated by us; Efnb2 $2^{\text {loxlox }}$ mice were obtained from The Max Planck Institute for Molecular Biomedicine; Nex-Cre mice were obtained from The Max Planck Institute of Experimental Medicine; Nestin-Cre mice were from The Jackson Laboratory. Efnb2 $2^{\text {H2BGFP }}$ and $E f n b 2^{\text {loxlox }}$ were on mixed genetic backgrounds; Nestin-Cre and Nex-Cre mouse lines were on a congenic C57B16JRj genetic background. To generate conditional mutants, Efnb2 ${ }^{\text {loxlox }}$ females were bred with Nestin-Cre; Efnb2 ${ }^{\text {loxlox }}$ males or Nex-Cre; $E f n b 2^{\text {loxlox }}$ males. Timed-pregnant mice were sacrificed by cervical dislocation with no prior anesthesia while embryos were euthanized by dissection with no prior anesthesia. These methods of euthanasia were approved by the appropriate Ethics Committee (APAFIS\#1289-2, 015,110,609,133,558 v5). Embryos were individually genotyped by PCR.

\section{Study design}

The study involved comparisons between embryos of two genotypes. The experimental unit is one embryo. Between 3 and 6 embryos from two different litters per genotype were analyzed by manual counting of molecular markers on paraffin sections. The researcher doing the manual counting was blinded to the genotypes. All data embryos were included in the analyses.

\section{Primary antibodies}

Satb2: Abcam, Mouse, ab51502, Paraffin: 1/150, Vibratome: $1 / 40$. 
Tbr1: Abcam, Rabbit, ab31940, Paraffin: 1/150, Vibratome: $1 / 100$.

Pax6: Covance, Rabbit, PRB-278P, 1/300.

Tbr2: Invitrogen, Rat, 14-4875-82, 1/100.

Cleaved Caspase 3: Cell signaling, Rabbit, 9661, 1/300.

Eph B2: R \& D Systems, Goat, AF467, WB: 1/1000.

Eph A4: Cell signaling, Rabbit, 8793, WB: 1/2000.

P-Eph B1-2: Abcam, Rabbit, ab61791, WB: 1/1000.

P-Eph A4: Gift from Greenberg's lab, Harvard medical school, Rabbit, WB: 1/1000.

\section{In situ hybridization}

In situ hybridization was performed on transverse vibratome sections as described previously [48].

\section{Protein extraction and Western blot}

For protein extraction, neocortex was dissected from E13.5 embryos in ice-cold PBS solution. Whole cell extracts were obtained by trituration in cold lysis buffer $(150 \mathrm{mM} \mathrm{NaCl}, 50 \mathrm{mM}$ Tris- $\mathrm{HCl} \mathrm{pH}$ 7.4, $0.5 \mathrm{mM}$ EDTA, $2 \mathrm{mM}$ Na3VO4, 1\% Nonidet P-40 (NP-40), $0.5 \mathrm{mM}$ EGTA and $0.1 \mathrm{mM}$ PMSF) supplemented by protease inhibitors (Roche) and incubation for $1 \mathrm{~h}$ on ice. Protein lysates were then vortexed, sonicated and centrifuged at $13,000 \mathrm{rpm}$ for $10 \mathrm{~min}$. Cleared lysates were used for Western blot analyses as described previously [41].

\section{In utero injections}

In utero injections were performed as described previously [41]. Briefly, timed-pregnant mice were anesthetized and uterine horns were exposed. $1 \mu \mathrm{g} / \mathrm{ml}$ human IgG or $1 \mu \mathrm{g} / \mathrm{ml}$ pre-clustered eB2-Fc (R\&D Systems) were injected in the lateral ventricle of E13.5 embryos neocortex. Body wall cavity and skin were sutured and embryos were allowed to develop normally for $48 \mathrm{~h}$ or until E18.5. Embryonic brains were collected at E15.5 or E18.5, fixed with $4 \%$ paraformaldehyde overnight at $4{ }^{\circ} \mathrm{C}$ and were processed for immunofluorescence staining as described below.

\section{Immunofluorescence}

Timed-pregnant mice were sacrificed by cervical dislocation; embryos were removed and dissected in ice-cold PBS. For immunohistochemistry, embryonic brains were collected and incubated in $4 \%$ PFA at $4{ }^{\circ} \mathrm{C}$ overnight. All brain samples were then either equilibrated in $70 \%$ ethanol and embedded in paraffin or sectioned on a vibratome. Vibratome sections were permeabilized and blocked with PBTA (2\% BSA, 2\% FBS, 1\% Tween20) for $2 \mathrm{~h}$ at room temperature. Sections were incubated overnight at $4{ }^{\circ} \mathrm{C}$ with primary antibodies diluted in PBTA and $1 \mathrm{~h} 30$ at room temperature with secondary antibodies diluted in PBS. Coronal paraffin sections $(5 \mu \mathrm{m})$ were rehydrated and incubated in Citrate buffer $\mathrm{pH} 6$ for
$45 \mathrm{~min}$ at $90^{\circ} \mathrm{C}$ for demasking. Immunostaining was then performed as described above.

\section{Image acquisition and quantification}

Microscope acquisitions were performed on an inverted SP8 (40x, TCS; Leica Biosystems) and entire hemispheres were acquired for each embryo. Cell counting was performed with the ImageJ software. To standardize cell counting, for each developmental stage, sections at similar rostral positions were selected, using anatomical landmarks (shape and size of the LGE, shape of the lateral ventricle, presence of the choroid plexus). For the loss of function experiments, at developmental stages E13.5 and E14.5, a single region of interest (dorsolateral) per hemisphere was used for manual counting. At later stages, 3 regions of interest (lateral, dorsal and medial) were used for manual counting. Except when otherwise stated, the data presented in the graphs is the average of the 6 regions of interest ( 2 hemispheres) for each embryo. For the gain of function experiments, a single region of interest (lateral or dorsal) per hemisphere was used for counting.

\section{Cell cycle analyses}

We used the protocol described by Martynoga [40]. Briefly, pregnant dam (E13.5) were injected with a single dose $(100 \mu \mathrm{l})$ of EdU $(10 \mu \mathrm{g} / \mathrm{ml})$, followed $1 \mathrm{~h} 30$ later by a single dose $(100 \mu \mathrm{l})$ of $\mathrm{BrdU}(10 \mu \mathrm{g} / \mathrm{ml})$. Embryos were collected $30 \mathrm{~min}$ later and processed for tissue sectioning and immunostaining to detect EdU+ cells, BrdU+ cells and Tbr2+ cells. We manually counted Tbr2- cells in the VZ corresponding to Pax6+ AP and Tbr2+ cells in a region of interest encompassing the central region of the neocortex. It is important to note that the EdU/BrdU double labeling method can be used to estimate cell cycle length only when analyzing populations of cycling cells, yet, Tbr2 remains expressed in newborn neurons which are no longer cycling. Thus, to circumvent this caveat we estimated the fraction of Tbr2+ cells that also express Tbr1 in both genotypes analyzed (Sup Figure 4) and assumed that these cells represent newborn neurons that have exited the cell cycle. We then used this to adjust the total number of Tbr2+ progenitors and obtain the number of P cells. We next counted L cells (P cells that are EdU+) and $\mathrm{S}$ cells ( $\mathrm{P}$ cells that are EdU + BrdU+). Length of the S-phase and length of the cell cycle were calculated using these numbers, as described in Fig. 3b and [40].

\section{Statistical analyses}

For experiments involving a single pair of conditions, statistical significance between the two sets of data were analyzed with an unpaired-t-test (Mann-Whitney) with Prism5 (GraphPad software). For datasets containing 
more than two samples, one-way analysis of variance (ANOVA) was used. Sample sizes of sufficient power were chosen on the basis of similar published research. For neuronal numbers, each dot on a graph represents a different embryo for which, depending on the stage, 3 ROI were manually counted, on 3-4 different sections. For progenitor numbers, each dot on a graph represent a single ROI averaged from 3 different sections for a single hemisphere. Between 3 and 6 embryos for each experimental conditions were analyzed. The value on the graph is the mean of these 3-4 points. Statistically significant differences are reported at ${ }^{*} P<0.05,{ }^{* * *} P<0.01$, ***: $P<0.005,{ }^{* * * * * * *} P<0.001$

\section{Supplementary information}

Supplementary information accompanies this paper at https://doi.org/10. 1186/s12861-020-00215-3.

Additional file 1: Figure S1. Validation of lox-Cre excision of Efnb2. Figure S2. Decreased numbers of neurons in Efnb2 mutants is not due to defective migration or increased apoptosis. Figure S3. Quantification of neuron numbers by type (Tbr1+ and Satb2+) at different developmental stages. Figure S4. Cell cycle analyses. Figure S5. In utero injection of EphB2-Fc.

\section{Abbreviations}

AP: Apical progenitors; BP: Basal progenitors; BrdU: Bromodeoxyuridine; BSA: Bovine serum albumin; CKO: Conditionnal knock-out; CP: Cortical plate; eB2-Fc: EphrinB2-Fc; EdU: 5-ethynyl-2'-deoxyuridine; DL: Deep layer; ISH: In situ hybridization; NP: Neural progenitors; PBS : Phosphate buffered saline; PBTA : Phosphate buffer Triton X-100; PFA: Paraformaldehyde; PMSF: Phenylmethylsulfonyl fluoride; ROI: Region of interest; SDS: Sodium dodecyl sulfate; SSC: Saline sodium citrate; SVZ: Subventricular zone; TBS : Tris buffered saline; UL: Upper layer; VZ: Ventricular zone

\section{Acknowledgements}

We thank Alain Vincent, Eric Agius and members of the Davy and Pituello teams for helpful discussions and comments on the manuscript. We acknowledge core support from the Imagery Platform of Toulouse and from the Anexplo animal facility.

\section{Authors' contributions}

AK and AD conceptualized the study, AK designed, performed and analyzed experiments and AD wrote the manuscript. MAF performed western-blot experiments. CA performed in utero injections and immunofluorescence experiments on paraffin sections. All authors contributed to analyzing experiments and to the editing of the manuscript. The author(s) read and approved the final manuscript.

\section{Funding}

This work was funded by ANR (Agence Nationale de la Recherche; ANR-15CE13-0010-01). Core funding was from the Université Paul Sabatier and the CNRS. The funders had no role in study design, data collection and analysis, decision to publish, or preparation of the manuscript.

\section{Availability of data and materials}

All data sets used and/or analyzed during the current study are available from the corresponding author on reasonable request.

\section{Ethics approval and consent to participate}

The manuscript was prepared following the ARRIVE guidelines and methodology.

Animal procedures were reviewed and approved by the Ethics Committee and Project Authorization \#001 accredited by the French Ministry of Research and Innovation. All procedures were in accordance with the
European Directive 2010/63. The methods of euthanasia were approved by the appropriate Ethics Committee (APAFIS\#1289-2,015,110,609,133,558 v5). The Max Planck Institute for Molecular Biomedicine and The Max Planck Institute of Experimental Medicine provided written consent to use mouse lines.

\section{Consent for publication}

Not applicable.

\section{Competing interests}

The authors declare that they have no competing interests.

Received: 13 January 2020 Accepted: 19 April 2020

Published online: 13 May 2020

\section{References}

1. Herculano-Houzel S, Mota B, Lent R. Cellular scaling rules for rodent brains. Proc Natl Acad Sci U S A. 2006:103(32):12138-43.

2. Greig LC, Woodworth MB, Galazo MJ, Padmanabhan H, Macklis JD. Molecular logic of neocortical projection neuron specification, development and diversity. Nat Rev Neurosci. 2013;14(11):755-69.

3. Lodato S, Shetty AS, Arlotta P. Cerebral cortex assembly: generating and reprogramming projection neuron diversity. Trends Neurosci. 2015;38(2): $117-25$

4. Harris KD, Shepherd GM. The neocortical circuit: themes and variations. Nat Neurosci. 2015;18(2):170-81.

5. Malatesta P, Hartfuss E, Gotz M. Isolation of radial glial cells by fluorescentactivated cell sorting reveals a neuronal lineage. Development. 2000;127(24): 5253-63.

6. Noctor SC, Flint AC, Weissman TA, Dammerman RS, Kriegstein AR. Neurons derived from radial glial cells establish radial units in neocortex. Nature. 2001;409(6821):714-20

7. Miyata T, Kawaguchi A, Okano H, Ogawa M. Asymmetric inheritance of radial glial fibers by cortical neurons. Neuron. 2001;31(5):727-41.

8. Noctor SC, Martinez-Cerdeno V, Ivic L, Kriegstein AR. Cortical neurons arise in symmetric and asymmetric division zones and migrate through specific phases. Nat Neurosci. 2004;7(2):136-44.

9. Miyata T, Kawaguchi A, Saito K, Kawano M, Muto T, Ogawa M. Asymmetric production of surface-dividing and non-surface-dividing cortical progenitor cells. Development. 2004;131(13):3133-45.

10. Haubensak W, Attardo A, Denk W, Huttner WB. Neurons arise in the basal neuroepithelium of the early mammalian telencephalon: a major site of neurogenesis. Proc Natl Acad Sci U S A. 2004;101(9):3196-201.

11. Vasistha NA, Garcia-Moreno F, Arora S, Cheung AF, Arnold SJ, Robertson EJ, Molnar Z. Cortical and clonal contribution of Tbr2 expressing progenitors in the developing mouse brain. Cereb Cortex. 2015:25(10):3290-302.

12. Hansen DV, Lui JH, Parker PR, Kriegstein AR. Neurogenic radial glia in the outer subventricular zone of human neocortex Nature. 2010:464:554-61.

13. Namba T, Huttner WB. Neural progenitor cells and their role in the development and evolutionary expansion of the neocortex. WIREs Dev Biol. 2017:6:e256.

14. Kawaguchi A. Temporal patterning of neocortical progenitor cells: how do they know the right time? Neurosci Res. 2019;138:3-11.

15. Herculano-Houzel S. The human brain in numbers: a linearly scaled-up primate brain. Front Hum Neurosci. 2009;3:31.

16. McConnell SK, Kaznowski CE. Cell cycle dependence of laminar determination in developing neocortex. Science. 1991:254:282-5.

17. Oberst P, Fievre S, Baumann N, Concetti C, Bartolini G, Jabaudon D. Temporal plasticity of apical progenitors in the developing mouse neocortex. Nature. 2019;573(7774):370-4.

18. Rodriguez M, Choi J, Park S, Sockanathan S. Gde2 regulates cortical neuronal identity by controlling the timing of cortical progenitor differentiation. Development. 2012;139:3870-9.

19. Parthasarathy S, Srivatsa S, Nityanandam A, Tarabykin V. Ntf3 acts downstream of Sip1 in cortical postmitotic neurons to control progenitor cell fate through feedback signaling. Development. 2014;141(17):3324-30.

20. Toma K, Kumamoto T, Hanashima C. The timing of upper-layer neurogenesis is conferred by sequential derepression and negative feedback from deep-layer neurons. J Neurosci. 2014;34:13259-76. 
21. Gerstmann K, Pensold D, Symmank J, Khundadze M, Hübner CA, Bolz J, Zimmer G. Thalamic afferents influence cortical progenitors via ephrin A5EphA4 interactions. Development. 2015;142:140-50.

22. Hatakeyama J, Shimamura K. The pace of neurogenesis is regulated by the transient retention of the apical Endfeet of differentiating cells. Cereb Cortex. 2019:29:3725-37.

23. Freret-Hodara B, Cui Y, Griveau A, Vigier L, Arai Y, Touboul J, Pierani A. Enhanced Abventricular proliferation compensates cell death in the embryonic cerebral cortex. Cereb Cortex. 2017;27(10):4701-18.

24. Kania A, Klein R. Mechanisms of ephrin-Eph signalling in development, physiology and disease. Nat Rev Mol Cell Biol. 2016;17:240-56.

25. Laussu J, Khuong A, Gautrais J, Davy A. Beyond boundaries: Eph/ephrin signaling in neurogenesis. Cell Adhes Migr. 2014;8:349-59.

26. Qiu R, Wang X, Davy A, Wu C, Murai K, Zhang H, Flanagan JG, Soriano P, Lu Q. Regulation of neural progenitor cell state by ephrin-B. J Cell Biol. 2008; 181:973-83.

27. North HA, Zhao X, Kolk SM, Clifford MA, Ziskind DM, Donoghue MJ. Promotion of proliferation in the developing cerebral cortex by EphA4 forward signaling. Development. 2009;136:2467-76.

28. Murai K, Qiu R, Zhang H, Wang J, Wu C, Neubig RR, Lu Q. Ga subunit coordinates with ephrin-B to balance self-renewal and differentiation in neural progenitor cells. Stem Cells. 2010;9:1581-9.

29. Arvanitis DN, Jungas T, Behar A, Davy A. Ephrin-B1 reverse signaling controls a post-transcriptional feedback mechanism in neural progenitors. Mol Cell Biol. 2010;30:2508-17.

30. Depaepe V, Suarez-Gonzalez N, Dufour A, Passante L, Gorski JA, Jones KR, Ledent C, Vanderhaeghen P. Ephrin signalling controls brain size by regulating apoptosis of neural progenitors. Nature. 2005;435:1244-50.

31. Park E, Kim Y, Noh H, Lee H, Yoo S, Park S. EphA/ephrin-a signaling is critically involved in region-specific apoptosis during early brain development. Cell Death Diff. 2013;20:169-80.

32. Kim Y, Park E, Noh H, Park S. Expression of EphA8-fc in transgenic mouse embryos induces apoptosis of neural epithelial cells during brain development. Dev Neurobiol. 2013;73:702-12.

33. Chen S, Bremer AW, Scheideler OJ, Na YS, Todhunter ME, Hsiao S, Bomdica PR, Maharbiz MM, Gartner ZJ, Schaffer DV. Interrogating cellular fate decisions with high-throughput arrays of multiplexed cellular communities. Nat Commun. 2016:7:10309.

34. Ashton RS, Conway A, Chinmay P, Bergen J, Kwang- $\|$ L, Shah P, Bissell M, Schaffer DV. Astrocytes regulate adult hippocampal neurogenesis through ephrin-B signaling. Nat Neurosci. 2012;15:1399-407.

35. Davy A, Soriano P. Ephrin-B2 forward signaling regulates somite patterning and neural crest cell development. Dev Biol. 2007;304:182-93.

36. Grunwald IC, Korte M, Adelmann G, Plueck A, Kullander K, Adams RH, Frotscher M, Bonhoeffer T, Klein R. Hippocampal plasticity requires postsynaptic ephrinBs. Nat Neurosci. 2004;7:33-40.

37. Tronche F, Kellendonk C, Kretz O, Gass P, Anlag K, Orban PC, Bock R, Klein R, Schutz $G$. Disruption of the glucocorticoid receptor gene in the nervous system results in reduced anxiety. Nat Genet. 1999:99:99-103.

38. Woodworth MB, Greig LC, Kriegstein AR, Macklis JD. SnapShot: cortical development. Cell. 2012;151(4):918 e911.

39. Goebbels S, Bormuth I, Bode U, Hermanson O, Schwab MH, Nave KA. Genetic targeting of principal neurons in neocortex and hippocampus of NEX-Cre mice. Genesis. 2006;44(12):611-21.

40. Martynoga B, Morrison H, Price DJ, Mason JO. Foxg1 is required for specification of ventral telencephalon and region-specific regulation of dorsal telencephalic precursor proliferation and apoptosis. Dev Biol. 2005; 283(1):113-27.

41. Fawal MA, Jungas T, Kischel A, Audouard C, lacovoni JS, Davy A. Cross talk between one-carbon metabolism, Eph signaling, and histone methylation promotes neural stem cell differentiation. Cell Rep. 2018;23(10):2864-73 e2867.

42. Lanctot AA, Guo Y, Le Y, Edens BM, Nowakowski RS, Feng Y. Loss of Brap results in premature G1/S phase transition and impeded neural progenitor differentiation. Cell Rep. 2017;20(5):1148-60.

43. Wu ZQ, Li D, Huang Y, Chen XP, Huang W, Liu CF, Zhao HQ, Xu RX, Cheng $M$, Schachner $M$, et al. Caspr controls the temporal specification of neural progenitor cells through notch signaling in the developing mouse cerebral cortex. Cereb Cortex. 2017;27(2):1369-85.

44. Jayaraman D, Bae BI, Walsh CA. The genetics of primary microcephaly. Annu Rev Genomics Hum Genet. 2018;19:177-200.
45. Eder D, Aegerter C, Basler K. Forces controlling organ growth and size. Mech Dev. 2017;144(Pt A):53-61.

46. Heuer K, Toro R. Role of mechanical morphogenesis in the development and evolution of the neocortex. Phys Life Rev. 2019;31:233-9.

47. Levy J, Haye D, Marziliano N, Casu G, Guimiot F, Dupont C, Teissier N, Benzacken B, Gressens P, Pipiras E, et al. EFNB2 haploinsufficiency causes a syndromic neurodevelopmental disorder. Clin Genet. 2018;93(6):1141-7.

48. Luxey M, Jungas T, Laussu J, Audouard C, Garces A, Davy A. Eph/ephrin-B1 forward signaling controls fasciculation of motor and sensory axons. Dev Biol. 2013;383:264-74.

\section{Publisher's Note}

Springer Nature remains neutral with regard to jurisdictional claims in published maps and institutional affiliations.
Ready to submit your research? Choose BMC and benefit from:

- fast, convenient online submission

- thorough peer review by experienced researchers in your field

- rapid publication on acceptance

- support for research data, including large and complex data types

- gold Open Access which fosters wider collaboration and increased citations

- maximum visibility for your research: over $100 \mathrm{M}$ website views per year

At BMC, research is always in progress.

Learn more biomedcentral.com/submissions 